\title{
Impact of different visualization devices on accuracy, efficiency, and dexterity in neurosurgery: a laboratory investigation
}

\author{
*Amol Raheja, MCh, Shashwat Mishra, MCh, Kanwaljeet Garg, MCh, Varidh Katiyar, MBBS, \\ Ravi Sharma, MCh, Vivek Tandon, MCh, Revanth Goda, MS, Ashish Suri, MCh, and \\ Shashank S. Kale, MCh
}

Department of Neurosurgery, All India Institute of Medical Sciences, New Delhi, India

OBJECTIVE Extracorporeal telescopes (exoscopes) have been the latest addition to the neurosurgeons' armamentarium, acting as a bridge between operating microscopes and endoscopes. However, to the authors' knowledge there are no published preclinical laboratory studies of the accuracy, efficiency, and dexterity of neurosurgical training for the use of $2 \mathrm{D}$ or $3 \mathrm{D}$ exoscopes compared with microscopes.

METHODS In a controlled experimental setup, 22 participating neurosurgery residents performed simple (2D) and complex (3D) motor tasks with three visualization tools in alternating sequence: a 2D exoscope, 3D exoscope, and microscope, using a block randomization model based on the neurosurgeons' prior training experience (novice, intermediate, and senior: $n=6,12$, and 4, respectively). Performance scores (PS; including error and efficiency scores) and dexterity scores (DS) were calculated to objectify the accuracy, efficiency, and finesse of task performance. Repeated measures ANOVA analysis was used to compare the PS, DS, and cumulative scores (CS) of candidates using the three visualization aids. Bland-Altman plots and intraclass correlation coefficients were generated to quantify intraobserver and interobserver agreement for DS. Subgroup analysis was performed to assess the impact of participants' prior training. A postexercise survey was conducted to assess the comfort level (on a 10-point analog scale) of the participants while using each visualization tool for performing the suturing task.

RESULTS PS, DS, and CS were significantly impacted by the visualization tool utilized for 2D motor tasks $(p<0.001$ for each), with the microscope faring better than the $2 D$ exoscope $(p=0.04)$ or $3 D$ exoscope $(p=0.008)$. The PS for the 3D object transfer task was significantly influenced by the visualization aid used $(p=0.007)$, with the microscope and 3D exoscope faring better than the 2D exoscope ( $p=0.04$ for both). The visualization instrument used significantly affected the DS and CS for the suturing task ( $p<0.001$ for both), with the microscope again scoring better than the 2D exoscope $(p<0.001)$ or $3 D$ exoscope $(p=0.005)$. The impact of the visualization aid was more apparent in participants with a shorter duration of residency (novice, $p=0.03$; intermediate, $p=0.0004$ ). Participants also felt the greatest operational comfort while working with a microscope, 3D exoscope, and 2D exoscope, in that order $(p<0.0001)$.

CONCLUSIONS Compared with $3 \mathrm{D}$ and $2 \mathrm{D}$ exoscopes, an operating microscope provides better dexterity and performance and a greater operational comfort level for neurosurgeons while they are performing 2D or 3D motor tasks. For performing complex 3D motor tasks, 3D exoscopes offer selective advantages in dexterity, performance, and operational comfort level over 2D exoscopes. The relative impact of visualization aids on surgical proficiency gradually weakens as the participants' residency duration increases.

https://thejns.org/doi/abs/10.3171/2020.10.FOCUS20786

KEYWORDS dexterity; 2D exoscope; microscope; visualization tools; neurosurgery; experimental design; 3D exoscope

ABBREVIATIONS BA = Bland-Altman; CS = cumulative score; DS = dexterity score; Ef = efficiency score; Er = error score; exoscope = extracorporeal telescope; HD = high definition; ICC = intraclass correlation coefficient; OR = operating room; PGY = postgraduate year; PS = performance score; $\mathrm{VITOM}=$ video telescopic operating microscope.

SUBMITTED August 31, 2020. ACCEPTED October 19, 2020.

INCLUDE WHEN CITING DOI: 10.3171/2020.10.FOCUS20786.

${ }^{*}$ A.R. and S.M. contributed equally to this work. 
$\mathrm{R}$ APID advancements in optical technology have revolutionized the field of neurosurgery over the past few decades with the introduction of high-definition (HD) and ultra-HD (4K and $8 \mathrm{~K}$ ) cameras, 2D and 3D neuroendoscopes, and next-generation microscopes with high magnification and integrated advanced features such as tumor fluorescence, intraoperative angiography, and heads-up neuronavigation display. ${ }^{1-3}$ Conventional microscopes provide good 3D visualization, but they are bulky and expensive, and occasionally an ergonomically challenging posture is needed for optimal visualization..$^{4-6}$ Neuroendoscopes, on the other hand, require a longer learning curve due to $2 \mathrm{D}$ vision and the need to use long instruments through small ports. ${ }^{3}$ Frequent soiling, fogging of the lens, and the inability to visualize the area proximal to the scope are other issues which discourage the use of neuroendoscopy in many delicate surgeries. ${ }^{5,6}$ To circumvent these issues without compromising the illumination and magnification required for neurosurgical procedures, extracorporeal telescope (exoscope) visualization systems have been conceptualized, which essentially are attempts to bridge the microscopy and neuroendoscopy domains and provide an affordable, portable, high-definition operative experience., 3

A 2D video telescopic operating microscope (VITOM; Karl Storz) was the first exoscope system described for use in neurosurgery and colposcopy, and later its potential was realized in other specialties as well. ${ }^{3,5,7}$ Next-generation 3D exoscopes like the VITOM-3D (Karl Storz), Zeiss KINEVO (Carl Zeiss AG), and Olympus ORBEYE (Olympus) systems circumvent the disadvantages of 2D exoscopes. ${ }^{3,8-10}$ Although there are a few clinical studies and a single cadaveric study demonstrating the effectiveness of 3D exoscopes, ${ }^{11}$ to our knowledge there has been no study to date comparing the impact of the $3 \mathrm{D}$ exoscope versus the $2 \mathrm{D}$ exoscope or the operating microscope on surgical proficiency and workflow efficiency. Hence, we conceptualized this controlled laboratory setting experimental study to objectively compare the impact of these visualization tools on accuracy, efficiency of task completion, and dexterity using a structured skills assessment module.

\section{Methods \\ Study Objective}

The primary objectives of the study were to assess the accuracy and efficiency of neurosurgical residents in completing simple (2D) and complex motor tasks (3D) in an allocated time interval, using three different types of visualization tools, a 2D exoscope (VITOM Karl Storz), a 3D exoscope (KINEVO Carl Zeiss), and an operating microscope (LEICA OH5, Leica Biosystems). The secondary objective was to assess the impact of each individual technology and the prior training (duration of residency experience) of the user on dexterity in performing simple motor tasks and suturing, which were assessed by four blinded experts using an analog scale. Additionally, we sought to understand participants' operational comfort level with each visualization technology by assessing their answers to a survey questionnaire.

\section{Study Design}

The study was designed as a preclinical laboratory evaluation (controlled experimental design) with enrollment of 22 neurosurgery residents in different stages of their residency training, who performed simple and complex motor tasks using the three visualization aids. All of the simple tasks were performed using fine-tip sketch pens attached rigidly to bayoneted instruments in order to simulate operative conditions. Participants were assigned to one of the following three groups based on the stage of their residency: novice $(n=6$, postgraduate year [PGY] 1-3 or equivalent), intermediate $(\mathrm{n}=12$, PGY 4-5 or equivalent), and senior ( $n=4$, PGY 6 or equivalent). A structured skills assessment module was designed and utilized to assess the superiority of each visualization tool in relation to the others. For valid comparison, the magnification for each tool was kept uniform and all exercises were performed using an adjustable chair and table to eliminate any ergonomic difficulty owing to the variability in the subject heights. Moreover, to reduce bias arising from learning and refinement of surgical skills, block randomization of the participants (within each subgroup based on stage of residency training) was performed to alter the sequence of use of visualization tools. All residents were familiar with the use of microscopes but were unfamiliar with the 2D and 3D exoscopes, as both were recently procured. These procedures ensured comparability of the data. All of the exercises were recorded in real time (Video 1).

VIDEO 1. Video showing the 2D motor, 3D object transfer, and suturing tasks. Copyright Amol Raheja. Used with permission. Click here to view.

Participants and observers did not receive any compensation. The study was not supported by any grant or any equipment donated by manufacturers.

\section{Simple Motor Tasks (2D)}

Four simple 2D motor tasks, which included drawing on calibrated graph paper with stipulated temporal and spatial protocols, were conceptualized to assess accuracy, efficiency/time taken to complete the tasks, and dexterity (as a surrogate for fine motor skill and finesse in hand-eye coordination) (Fig. 1). Accuracy was assessed by calculating demerits (or errors) for each instance of violation of the shaded area for tasks A and C, and overshooting, undershooting, or missing of dots for tasks B and D (Video 1). The cumulative number of errors were calculated for each task separately to assess inaccuracy. Efficiency (for all simple tasks A-D) was calculated by comparing the time taken to finish each task within the prescribed cumulative time limit of 6 minutes. This was also a surrogate evaluation for the speed of 2D task performance based on the impact of each visualization tool on hand-eye coordination. The efficiency score (Ef) and error score (Er) were then calculated as proportions of the scores of the candidate with maximum efficiency (minimum time taken) or minimum errors per task across all visualization aids respectively (Supplemental Table 1). A performance score (PS) was then calculated after penalizing half of the Ef using the Er (Table 1). 


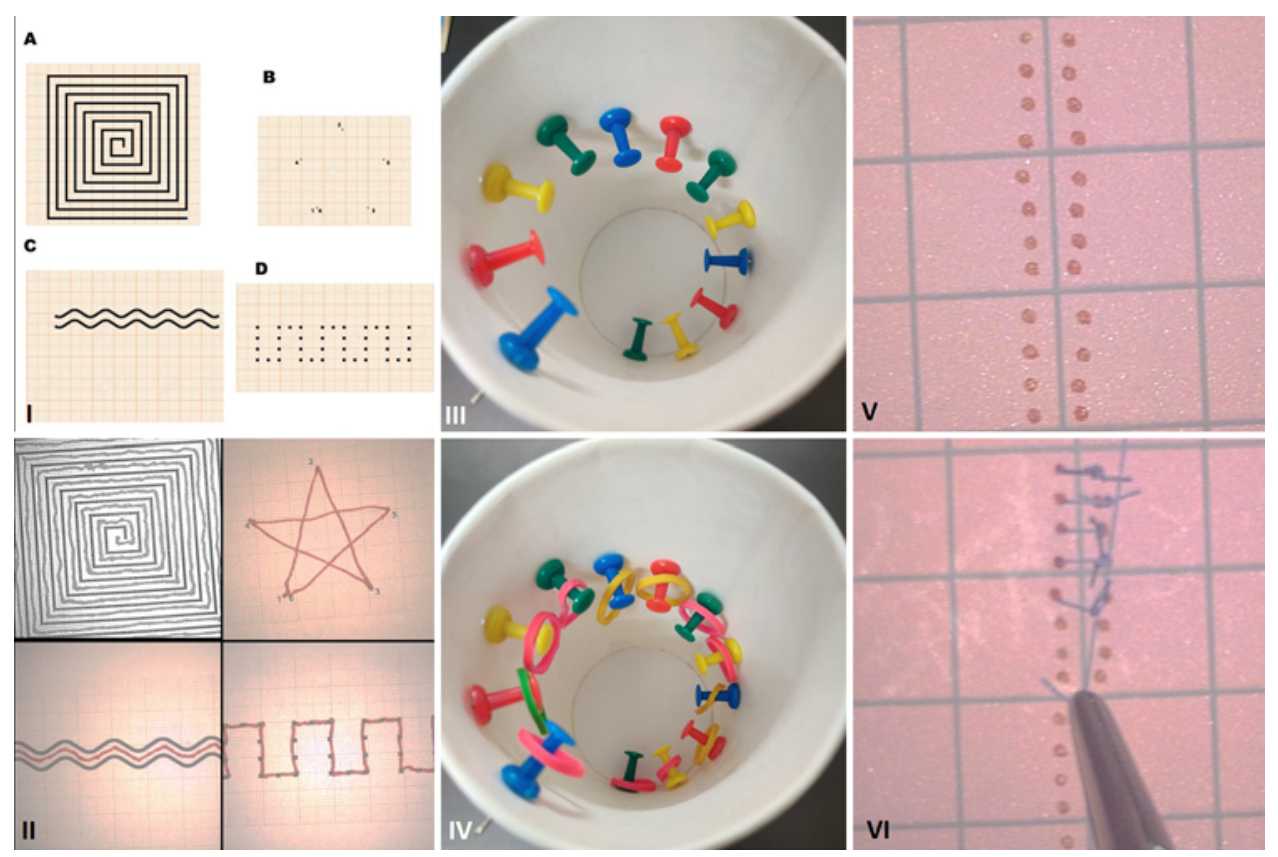

FIG. 1. Representative images of the various tasks. I: 2D motor task. II: 2D motor task completed by a participant. III: 3D object transfer task. IV: 3D object transfer task being performed by the participant. V: Suturing task. VI: Suturing task being performed by the participant.

\section{Complex Motor Tasks (3D)}

To assess depth perception under simulated operating conditions, two complex motor tasks were designed to assess accuracy, efficiency, and dexterity. The first task (3D object transfer) was the use of a bayoneted instrument to transfer rubber rings to prefixed drawing pins placed in a $360^{\circ}$ spiral arc in a disposable paper cup (Fig. 1, Video 1). The time taken to complete this task (as a surrogate for efficiency) was noted along with the number of rings hanging appropriately over the drawing pins and the number of rings which fell within the cup (as a surrogate for accuracy). All the scores were then calculated as outlined in the 2D motor task (Table 1). The second complex motor task was microsuturing along predefined entry and exit points on a silastic sheet using 5-0 Vicryl (3 surgical knots) during an allotted time of 7 minutes (Fig. 1, Video 1). The numbers of sutures completed were calculated (as a surrogate for speed and efficiency of task performance), along with demerits (or errors) calculated for inaccuracy in piercing the marked entry or exit point while taking the suture, and correct placement of the suture knot over the midline (as a surrogate for accuracy). All the scores were calculated for the suturing task as well in a similar fash- ion. The Er was calculated using the error rate per suture (i.e., error rate per unit work, Supplemental Table 1).

\section{Protocol for Dexterity Score Assessment}

In order to assess fine motor function and finesse in hand-eye coordination using different visualization technologies, test runs were performed by the authors the day before the actual assessment workshop to enable them to understand the difficulty levels of each allocated task for assessing dexterity based on the scoring weight of each component of the $2 \mathrm{D}$ motor tasks and the suturing task. A consensus for allocating 5:3:1:1 weighted scores for the four 2D motor tasks (Video 1) was arrived at for all assessments of 2D motor tasks (maze drawing on graph paper shown in Fig. 1). A dexterity score (DS) for each 2D task was awarded based on the assessment of the smoothness/ fluency of the task performance, geometrical symmetry, aesthetic appeal, and degree of precision in carrying out the tasks per the defined protocol without undue violations. For the suturing task, the DS was awarded based on the proficiency of the suturing technique, handling of suture material, surgical finesse, precision of travers-

TABLE 1. Scoring system used for 2D motor, 3D object transfer, and suturing tasks

\begin{tabular}{cll}
\hline Task & \multicolumn{1}{c}{ Ef } & Er \\
\hline 3D motor & Minimum time taken/time taken by candidate & Errors made by candidate/maximum errors made \\
\hline Suturing & Sutures placed by candidate/maximum sutures placed & (Errors/suture made by candidate)/(maximum errors/sutures made) \\
\hline
\end{tabular}

$\mathrm{PS}=\mathrm{Ef} \times(1-\mathrm{PF} \times \mathrm{Er}) \times 100 ; \mathrm{PF}$ (penalization factor) $=0.5$; PF signifies the proportion of the Ef to be penalized with the $\mathrm{Er}$.

$\mathrm{DS}=($ candidate DS/maximum DS $) \times 100$.

$\mathrm{CS}=(\mathrm{PF}+\mathrm{DS}) / 2$. 

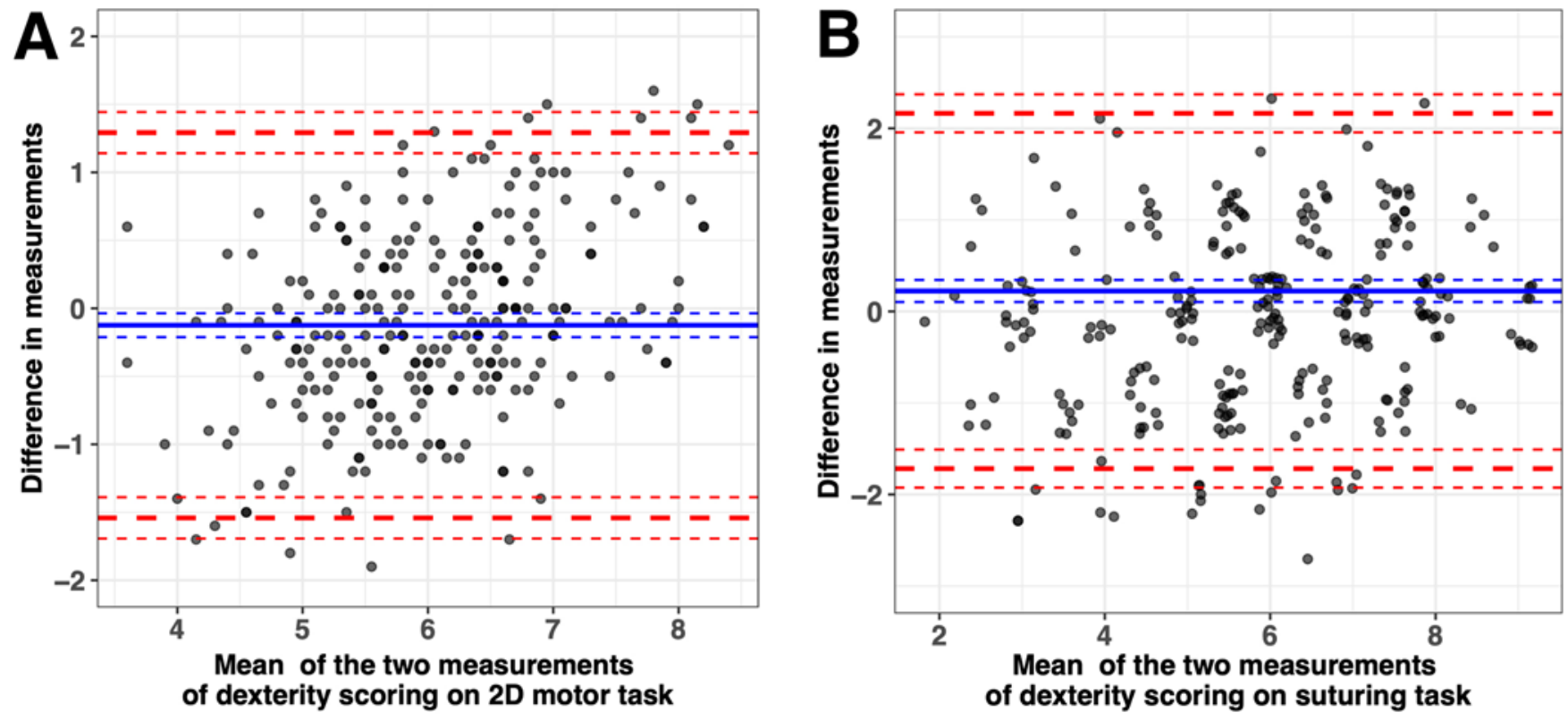

FIG. 2. A: BA plot showing intraobserver variability in the dexterity scoring of the $2 \mathrm{D}$ motor task. B: BA plot showing intraobserver variability in the dexterity scoring of the suturing task.

ing through entry and exit points, knotting technique, and final position of suture knots. To minimize assessment bias, four experts (faculty members with at least 5 years of postresidency experience) assessed the video recordings of the 2D motor tasks (Video 1) and the suturing task, and graded them on a scale of 1 to 10 . The assessors were blinded to the visualization tools used and the identity of the subjects. The same exercise was repeated after 24 hours with a randomly rearranged video sequence, to minimize intraobserver variability. Scoring was done on separate Excel sheets, and the average cumulative scores of both assessments were calculated for the 2D motor task and the suturing task to ascertain the final DS (Table 1). The interobserver variability and intraclass correlation coefficients (ICCs) were calculated to ascertain the reliability of the scoring system used (Fig. 2 and Table 2). A cumulative score (CS) was then awarded to each candidate using the mean of their PS and DS (Table 1).

\section{Participant Feedback}

A postexercise survey was conducted (Supplemental Questionnaire) to rate the level of operational comfort perceived by each participant using each visualization tool on a 10-point analog scale for the suturing task.

\section{Statistical Analysis}

The data were entered using Microsoft Excel 2016 and statistical analysis was done using R software (R Foundation for Statistical Computing) employing the packages ggplot $2^{26}$ and rstatix. One-way repeated measures ANOVA was used to compare the performance, dexterity, and cumulative scores of candidates using the three visualization aids, since repeated observations were made for the same group of participants. Bland-Altman (BA) plots were generated to estimate the limits of agreement between the two evaluations of the same subject by the same observer for

TABLE 2. Summary of interobserver agreement and ICCs for the dexterity scoring for both suturing and $2 \mathrm{D}$ motor tasks

\begin{tabular}{ccccc}
\hline \multirow{2}{*}{ Task } & \multicolumn{3}{c}{ Interobserver Agreement, \% } & \\
\cline { 2 - 4 } & Absolute (same score) & w/in \pm 1 Unit & w/in \pm 2 Units & Type ICC 3k $(95 \%$ CI) \\
\hline Microsuturing & 21.2 & 83.3 & 100 & $0.98(0.97-0.98)$ \\
\hline 2D motor task & & & \\
\hline A & 12.1 & 63.6 & 97 & $0.94(0.92-0.96)$ \\
\hline B & 12.1 & 62.1 & 98.5 & $0.93(0.91-0.95)$ \\
\hline C & 13.6 & 71.2 & 95.5 & $0.91(0.88-0.94)$ \\
\hline D & 12.1 & 75.8 & 98.5 & $0.91(0.88-0.94)$ \\
\hline CS & 66.7 & 98.5 & $0.95(0.94-0.97)$ \\
\hline * Used when quantitative measurements are done on units that are organized into groups to assess how strongly the \\
units or groups resemble each other/correlate.
\end{tabular}



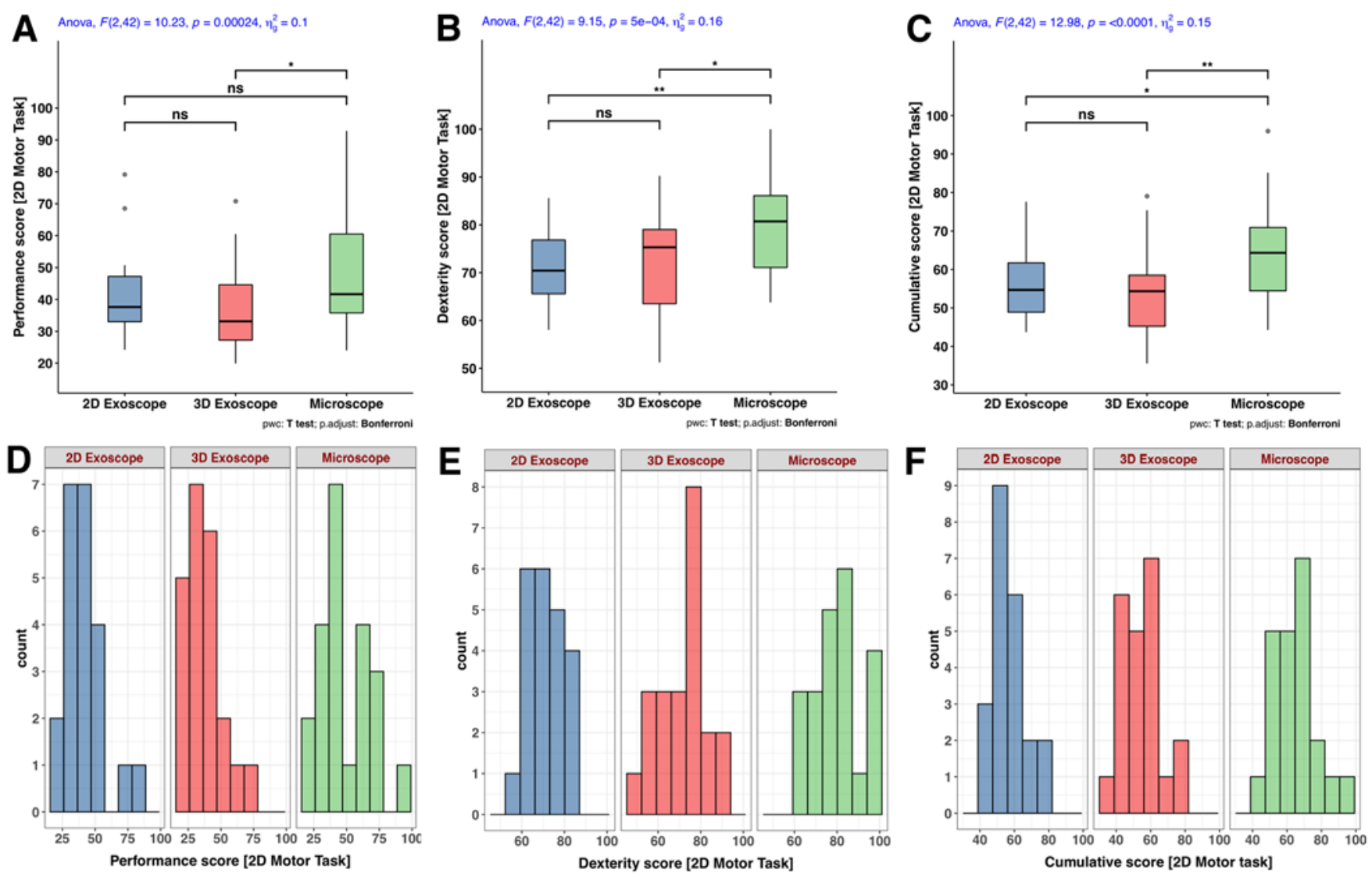

FIG. 3. A-C: Box-and-whisker plots for comparison of scoring for 2D motor tasks among the different visualization aids, PS (A), DS (B), and CS (C); ns = not significant; ${ }^{*},{ }^{* *}$ significant (level of significance increases with the number of asterisks). D-F: Histograms showing the scoring for the $2 \mathrm{D}$ motor tasks among the different visualization aids, PS (D), DS (E), and CS (F).

the given task. The interobserver agreement was quantitated using the ICC (expressed as the coefficient of difference, $95 \%$ confidence interval). For all statistical tests, a p value $<0.05$ was considered statistically significant.

\section{Results}

\section{Intraobserver and Interobserver Variability: Dexterity Score Assessment}

The intraobserver agreement was assessed through a BA analysis of the two scores awarded to a particular activity by the same observer on the repeated video assessments. The limits of agreement for the dexterity scoring for 2D motor tasks was determined to range from 1.3 (95\% CI 1.14 to 1.44 ) to -1.5 (95\% CI, -1.7 to -1.4$)$. Corresponding limits for the suturing dexterity assessment were $2.2(95 \%$ CI 1.96 to 2.37 ) and -1.7 (95\% CI -1.9 to -1.5$)$ (Fig. 2A and $\mathrm{B})$. The ICC calculated for estimating the agreement between the blinded observers evaluating a particular task was higher than 0.90 across all modules of assessment, demonstrating excellent interrater reliability (Table 2).

\section{Impact of Visualization Tool \\ 2D Motor Task}

The PS was found to be significantly related to the visualization aid used ( $<<0.001$, Fig. 3A and D). On pairwise comparison, the PS was significantly better with the use of the microscope than of the 3D exoscope $(p=0.04)$, but not better than the PS with the use of the 2D exoscope (p $=0.34$ ). In comparison, there was a significant difference in the DS across the modalities $(\mathrm{p}<0.001$, Fig. 3B and $\mathrm{E})$, with subjects faring better with the microscope than with the 3D exoscope $(p=0.01)$ or the $2 \mathrm{D}$ exoscope $(\mathrm{p}=$ 0.009 ). However, no difference was observed between $2 \mathrm{D}$ and $3 \mathrm{D}$ exoscopes $(\mathrm{p}=1.00)$. Consequently, a significant difference was also observed among the three visualization aids in the subject CS scores $(\mathrm{p}<0.001$, Fig. $3 \mathrm{C}$ and F). Similarly, on pairwise post hoc comparisons, a significant difference was found between the microscope and the $3 \mathrm{D}$ exoscope $(p=0.008)$ or $2 \mathrm{D}$ exoscope $(p=0.04)$, but no apparent difference was noticed between the 3D exoscope and the 2D exoscope $(\mathrm{p}=1.00$, Table 3$)$.

\section{D Object Transfer Task (ring transfer in 3D space)}

The PSs for the 3D object transfer task were found to be significantly different according to the visualization aid used ( $\mathrm{p}=0.007$, Fig. 4A and B). On pairwise comparison, PS was found to be significantly lower in subjects using the $2 \mathrm{D}$ exoscope than in subjects using the 3D exoscope or microscope ( $p=0.04$ for both). However, no significant difference in PS was seen in the comparison between the microscope and 3D exoscope $(\mathrm{p}=1.00$, Table 3$)$. 
TABLE 3. Summary of participant scores obtained in the various tasks

\begin{tabular}{|c|c|c|}
\hline Task \& Modality & Score & p Value* \\
\hline \multicolumn{3}{|l|}{ 2D motor task } \\
\hline \multicolumn{3}{|l|}{ Performance } \\
\hline 2D exoscope & $41.0 \pm 13.2$ & \multirow{3}{*}{$<0.001$} \\
\hline 3D exoscope & $36.8 \pm 12.9$ & \\
\hline Microscope & $48.1 \pm 17.7$ & \\
\hline \multicolumn{3}{|l|}{ Dexterity } \\
\hline 2D exoscope & $71.3 \pm 7.9$ & \multirow{3}{*}{$<0.001$} \\
\hline 3D exoscope & $71.5 \pm 11.2$ & \\
\hline Microscope & $80.8 \pm 11.4$ & \\
\hline \multicolumn{3}{|l|}{ Cumulative } \\
\hline 2D exoscope & $56.1 \pm 9.2$ & \multirow{3}{*}{$<0.001$} \\
\hline 3D exoscope & $54.2 \pm 10.8$ & \\
\hline Microscope & $64.5 \pm 12.5$ & \\
\hline \multicolumn{3}{|c|}{ 3D object transfer task } \\
\hline \multicolumn{3}{|l|}{ Performance } \\
\hline 2D exoscope & $41.5 \pm 17.4$ & \multirow{3}{*}{0.007} \\
\hline 3D exoscope & $52.7 \pm 19.4$ & \\
\hline Microscope & $53.3 \pm 17.6$ & \\
\hline \multicolumn{3}{|l|}{ Suturing task } \\
\hline \multicolumn{3}{|l|}{ Performance } \\
\hline 2D exoscope & $47.5 \pm 15.1$ & \multirow{3}{*}{0.104} \\
\hline 3D exoscope & $48.7 \pm 13.6$ & \\
\hline Microscope & $54.1 \pm 15.4$ & \\
\hline \multicolumn{3}{|l|}{ Dexterity } \\
\hline 2D exoscope & $37.6 \pm 13.8$ & \multirow{3}{*}{$<0.001$} \\
\hline 3D exoscope & $49.9 \pm 21.1$ & \\
\hline Microscope & $73.2 \pm 18.7$ & \\
\hline \multicolumn{3}{|l|}{ Cumulative } \\
\hline 2D exoscope & $42.5 \pm 12.8$ & \multirow{3}{*}{$<0.001$} \\
\hline 3D exoscope & $49.3 \pm 15.5$ & \\
\hline Microscope & $63.7 \pm 15.4$ & \\
\hline
\end{tabular}

Mean values are presented \pm SD.

* Repeated measures ANOVA.

\section{Suturing Task}

The PS for the suturing task was not found to be significantly affected by the visualization aid used, although a trend was evident ( $p=0.10$, Fig. 5A and D). However, there was a significant difference in the DS across the modalities ( $p<0.001$, Fig. 5B and E), with the microscope faring better than both the 3D exoscope $(\mathrm{p}<0.001)$ and the $2 \mathrm{D}$ exoscope $(\mathrm{p}<0.001)$. A trend was observed in favor of the $3 \mathrm{D}$ exoscope over the $2 \mathrm{D}$ exoscope, but this trend failed to reach statistical significance $(\mathrm{p}=0.08)$. Similar to the DS, the CS for the suturing task was also found to be significantly higher with the use of the microscope $(\mathrm{p}<0.001$, Fig. $5 \mathrm{C}$ and $\mathrm{F}$ ) than with either of the other modalities, i.e., the $2 \mathrm{D}$ exoscope $(\mathrm{p}<0.001)$ or $3 \mathrm{D}$ exoscope $(\mathrm{p}=0.005)$. However, for the suturing task there was no apparent difference in PS between the 3D exoscope and 2D exoscope $(\mathrm{p}=0.40$, Table 3$)$.

\section{Impact of Prior Training}

Interestingly, we observed a significant impact of the visualization modality when comparing the suturing tasks for the novice $(\mathrm{p}=0.03)$ and intermediate groups $(\mathrm{p}=0.0004)$, with significantly better performance with the microscope (Fig. 6A). However, the visualization tools used did not significantly alter the CS for suturing tasks in the senior group $(\mathrm{p}=0.40)$. On pairwise comparisons, the difference in performance was significantly apparent between the microscope and 2D exoscope groups for both the novice and intermediate subgroups $(\mathrm{p}=0.04$ and $\mathrm{p}=$ 0.0002 , Fig. 6A). In comparison, there was a similar trend for the comparison of the use of the microscope and 3D exoscope by both the novice and intermediate groups $(\mathrm{p}=$ 0.06 and $p=0.05$, respectively, Fig. 6A). On the contrary, stratifying the participants based on their prior training within each visualization modality group, no significant difference was observed in the performance of the suturing tasks among the participants with different training levels ( $p>0.05$ for each intragroup comparison, Fig. 6B).

\section{Participant Survey Analysis}

For the suturing task, the participants reported greater operational comfort in using the microscope than the 3D exoscope $(p<0.01)$ or the 2D exoscope $(p<0.0001$, Fig. $6 \mathrm{C})$. Furthermore, the 3D exoscope offered better participant comfort scores in comparison to the 2D exoscope ( $p$ $<0.001$, Fig. 6C). On analyzing the data further, for participant operational comfort levels the superiority of the microscope over the 2D and 3D exoscopes was evident in all aspects of the suturing tasks, i.e., grasping the needle, grasping the suture ends, cutting the suture ends, and tying the knot $(\mathrm{p}<0.0001$ for each, Fig. 6D and Supplemental Table 2).

\section{Discussion}

Illumination and magnification are the two vital cornerstones upon which neurosurgery has evolved over time. This study has objectively revealed the impact of different visualization devices on accuracy, efficiency, and dexterity in the performance of tasks by neurosurgery residents at different stages of training.

\section{Visualization and Hand-Eye Coordination}

The changing trend of adopting "sight-line" technology over a "through-the-lens" interface has propelled the paradigm shift from microscope- to exoscope-based visualization in the past decade. ${ }^{5,12}$ Rapid transition to first-generation exoscopes was constrained by their 2D visualization, poorer hand-eye coordination, inadequate illumination in the depths of field, and an associated steep learning curve., ${ }^{4,13,14}$ These attributes and limitations of 2D technology are also highlighted in our experimental study, where neurosurgery residents using a 2D exoscope had poorer hand-eye coordination and level of operational comfort (Fig. 5C and D) leading to lower performance, dexterity, and cumulative scores compared to residents using a 3D exoscope (Figs. 2-4). Initial reports by Mamelak et al. of the clinical use of the VITOM utilizing a 3-chip HD camera suggested that the image quality was 

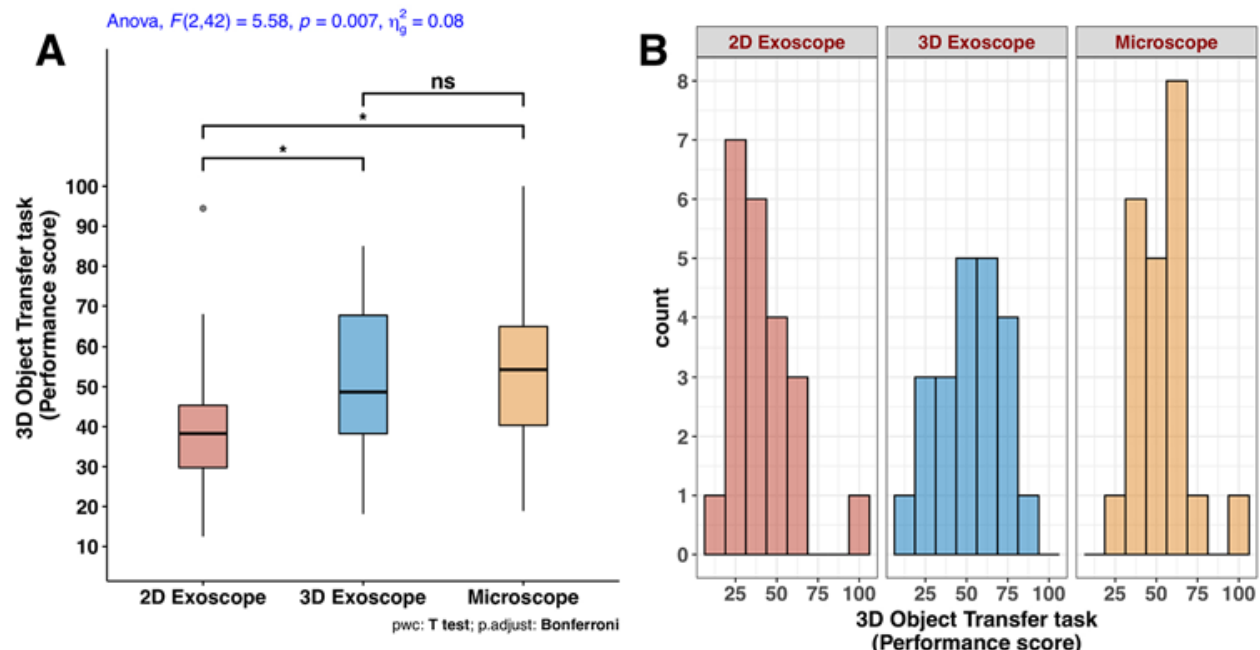

FIG. 4. A: Box-and-whisker plot for comparison of scoring for the 3D object transfer task among the different visualization aids; *significant. B: Histogram showing the scoring for the 3D object transfer task among the different visualization aids.

similar to that of an operating microscope; however, the absence of true 3D vision hampered operative proficiency in cranial cases and offered a longer learning curve. ${ }^{10}$ Similar sentiments were resonated by other clinical case series as well, especially for surgeries performed through narrow operative corridors utilizing tubular retractors..$^{5-17}$
Our observations using complex 3D motors tasks were concurrent, where lack of appropriate depth perception with the use of a 2D exoscope was a major hindrance in smooth performance of the tasks by participants (Figs. 3 and 4).

The next-generation exoscopes incorporated 3D 4K-HD
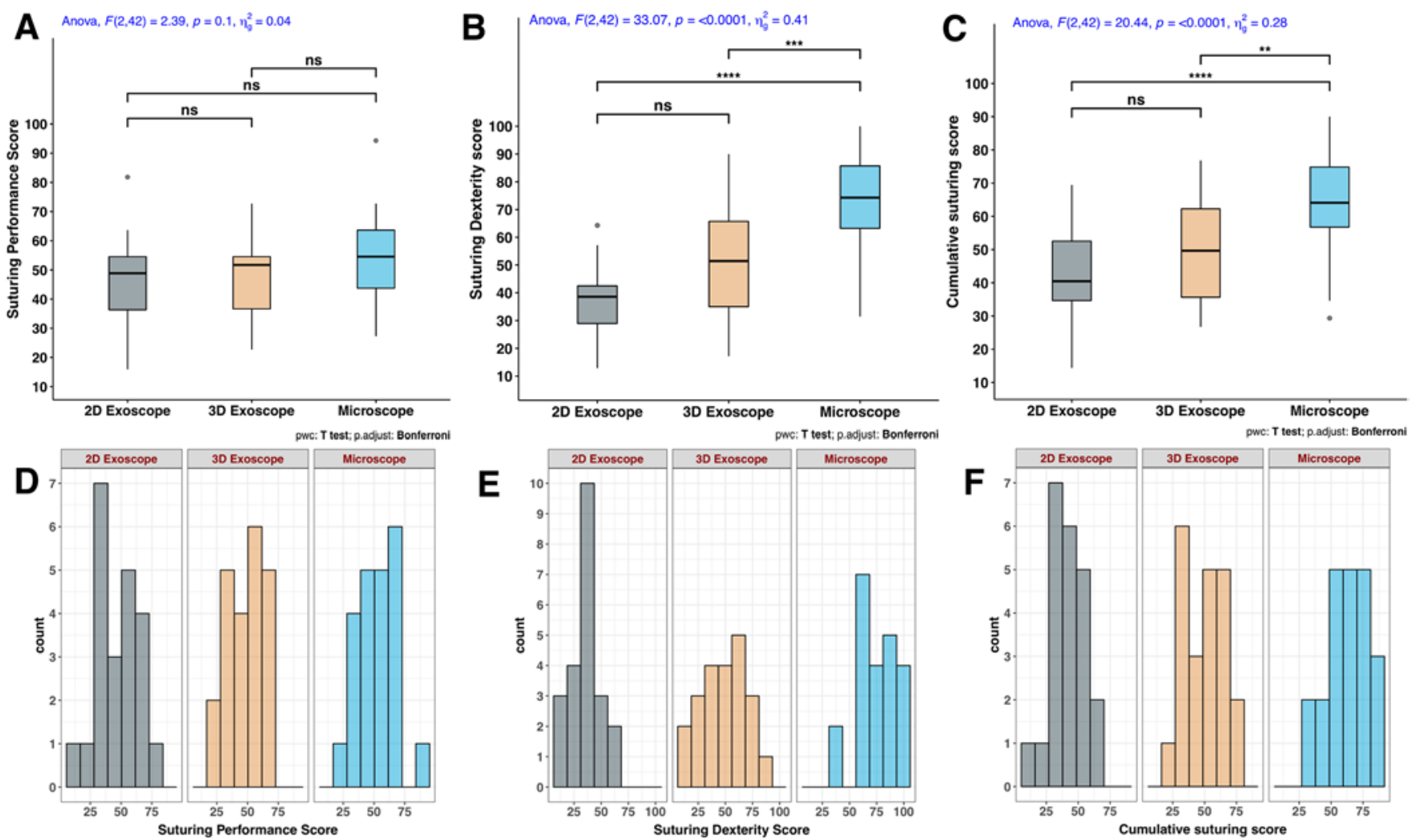

FIG. 5. A-C: Box-and-whisker plot for comparison of scoring for the suturing task among the different visualization aids, PS (A), DS (B), and CS (C); ${ }^{* *}{ }^{* * *},{ }^{* * * *}$ significant (level of significance increases with the number of asterisks). D-F: Histograms showing the scoring for the suturing task among the different visualization aids, PS (D), DS (E), and CS (F). 

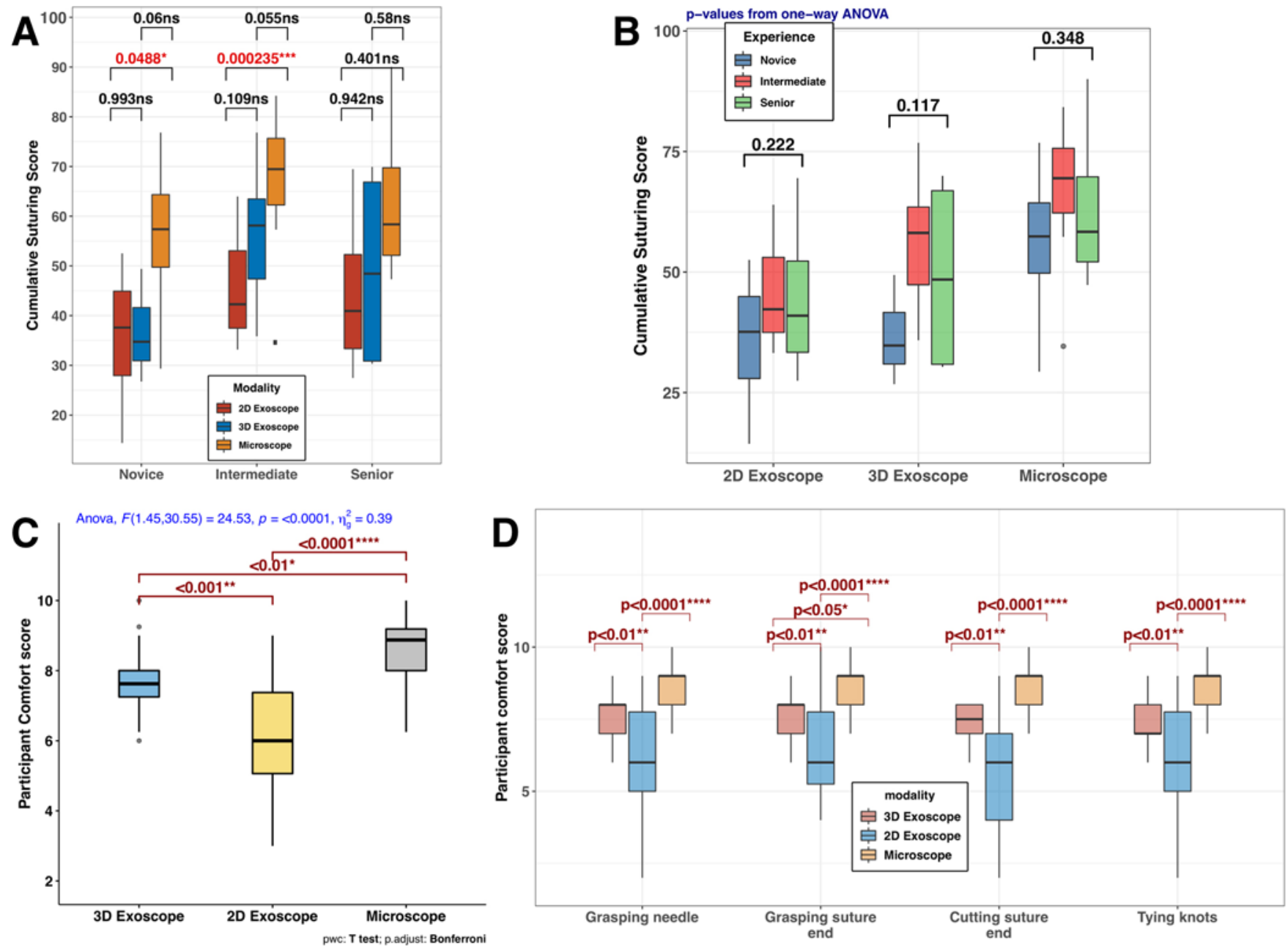

FIG. 6. A: Box-and-whisker plot for comparison of cumulative suturing score among different visualization aids for participants of each training level. B: Box-and-whisker plot for comparison of cumulative suturing score among participants with different levels for each visualization aid. $C$ and D: Box-and-whisker plot for comparison of the perceived level of operational comfort of participants among different visualization aids for the suturing task, complete task (C) and individual task (D) components; ${ }^{*},{ }^{* *},{ }^{* * *}$, ****significant (level of significance increases with the number of asterisks).

technology to overcome the shortcomings of its older counterparts, which lacked stereopsis, and offer clinical use options for a wider range of complex neurosurgical procedures; however, the efficacy of these instruments in high-precision cranial skull base and vascular procedures is yet to be uniformly validated..$^{2,9,18}$ In comparison to a standard operating microscope, a 3D exoscope offers a larger operative corridor and higher focal length range and depth of field, and it more closely approaches an immersive surgical experience. A 3D exoscope also offers all the operating team members the same HD view as that afforded to the primary operator, thereby potentially enabling more efficient operating room (OR) workflow by allowing technicians, anesthesiologists, and OR support staff to be more engaged in the surgical procedure and better anticipate surgeons' needs. ${ }^{6,19,20}$ However, the operating microscope still remains the gold standard for most neurosurgical procedures and centers across the world as it has stood the test of time, due to its innate ability to provide excellent stereoscopic vision, a synchronized inline 3D visual perception feedback loop, operative maneuverability, and validated clinical effectiveness in highprecision skull base and vascular surgeries. ${ }^{5,6}$ Along similar lines, using our experimental design, we demonstrated that in comparison to $3 \mathrm{D}$ exoscopes, an operating microscope enables the user to work with better dexterity, performance, and operational comfort (Fig. 5C and D) while performing $2 \mathrm{D}$ motor tasks and suturing tasks (Figs. 2 and 4). The apparent difference observed could be due to a more natural visual experience and refined stereoscopic optical image quality rendered by binocular lenses in operating microscopes compared to virtual and digital 3D images created by 3D exoscopes. However, prior experience of microscope use in clinical practice by participants may have affected this comparison with 2D/3D exoscope technologies, which were novel and unfamiliar for them at the time of this study. 


\section{Ergonomics, Versatility, and Body Mechanics}

Extracorporeal telescopes (or exoscopes) have a significant edge over operating microscopes with regard to ergonomics, versatility, and smooth conduct of OR workflow, by virtue of their lighter and petite design, portability, higher depth of field with limited need for readjustment, enhanced instrument maneuverability, smooth passage of instruments in and out of operative field, and excellent surgeon physical comfort while working in a neutral posture. However, most iterations of exoscopes suffer from inbuilt mechanical constraints related to repositioning, refocusing, and magnification using the scope holder arm, which hinders the hassle-free workflow dynamics. Integration of pneumatic arms may serve as a potential breakthrough in efficient utilization of present-generation exoscopes., ${ }^{1,2,14,21}$

We observed similar findings during our experimental study, especially with regard to the ergonomic advantages offered by 2D/3D exoscopes, which enabled the smooth conduct of task workflow due to their lightweight and compact design. As mentioned above, however, repositioning and refocusing the 2D/3D exoscopes had its own set of challenges, which can be especially problematic where rapid readjustments in visual-field/focus/magnification are required, as in managing intraoperative hemorrhage, brain bulge, etc. ${ }^{1,14,22}$ Yet another potential limitation for widespread use of 3D exoscopes is the risk of vestibular symptoms (nausea, vomiting, dizziness, etc.) from using polarized 3D glasses for the long periods that are often required for complex neurosurgical procedures. ${ }^{3,6}$ None of the participants reported the above symptoms, probably due to the short (15 minutes) duration of exposure on the 3D exoscope station. Similarly, we could not ascertain the differences in the level of physical comfort between microscope and 2D/3D exoscope groups, as the designed tasks were of short duration and included working in a neutral body posture. Technological advances in the field of optics, imaging sensors, virtual and augmented reality devices, and novel 3D interfaces could redefine intraoperative experience, user satisfaction, and surgical ergonomics in future..$^{5,23}$ Although preliminary data support favorable economics for exoscopes over operating microscopes, the long-term cost-effectiveness of exoscopes remains to be validated. ${ }^{7,8,20}$

\section{Training and Education}

The next-generation 3D exoscope offers an excellent platform for training and education of residents, fellows, and nurses, as it allows 3D 4K-HD visualization of operative fields uniformly to all the team members, in contrast to an operative microscope, where the highest-quality images with binocular vision and 3D perception are reserved for operating/assisting surgeons. The use of these instruments can augment the intuitive understanding of anatomical details by providing a real-time 3D view on a $4 \mathrm{~K}$ screen. ${ }^{5,6,9}$ In addition, working on a 3D platforms helps to create a roadmap for surgical procedures by heightening the sensory inputs, akin to virtual and augmented reality devices, thereby shortening the learning curve of any procedure. ${ }^{24,25}$ Interestingly, we observed a significantly higher impact of visualization modality upon the suturing performance for participants in early stages of their residency (novice and intermediate), with the microscope faring significantly better than the 2D exoscope and showing a similar trend with the 3D exoscope as well (Fig. 5A). However, this impact was attenuated in the senior group of participants (PGY 6 or equivalent, Fig. 5A), possibly due to a smaller cohort, greater refinement of surgical skills, and a degree of familiarity with the similar technologies of 2D/3D neuroendoscopy during their entire residency tenure. In other words, the differences in the relative impacts of visualization aids on surgical proficiency gradually weaken as the participants' residency duration increases. ${ }^{3}$

\section{Study Limitations}

The innate hand-eye coordination of each study participant, irrespective of the residency stage or visualization tool, may have impacted the observations. The variability in the manufacturers for the three technologies used at the three workstations may have also introduced bias in the data collected. Last, the test-retest variability was not accounted for in this study as the data collection was performed over a single session and due to logistical constraints was not repeated.

\section{Conclusions}

The operating microscope facilitates dexterity and provides greater operational comfort that enhances performance of $2 \mathrm{D} / 3 \mathrm{D}$ motor tasks in comparison to $3 \mathrm{D} / 2 \mathrm{D}$ exoscopes. The 3D exoscope offers selective advantages in dexterity, performance, and operational comfort levels over the 2D exoscope while performing complex 3D motor tasks. The relative impact of the visualization aid on surgical proficiency gradually weakens as the residency duration of the user increases.

\section{Acknowledgments}

We thank Ms. Paramjeet Kaur and Ms. Rani Beji, our senior nursing officers, and their team for logistical support; Mr. Nam Prasad, our senior technician, and his team for technical assistance in recording; and the staff from our "neurosurgery skills training facility" for extending their support. We thank Mr.

Akshay Raheja and Mr. Syed Atif Hussain for their assistance in creating the graphic designs for the simple motor tasks in Fig. 1 and Video 1.

\section{References}

1. Bauer AM, Rasmussen PA, Bain MD. Initial single-center technical experience with the BrainPath system for acute intracerebral hemorrhage evacuation. Oper Neurosurg (Hagerstown). 2017;13(1):69-76.

2. Mamelak AN, Drazin D, Shirzadi A, et al. Infratentorial supracerebellar resection of a pineal tumor using a high definition video exoscope (VITOM®). J Clin Neurosci. 2012;19(2): 306-309.

3. Raheja A, Kalra R, Couldwell WT. Three-dimensional versus two-dimensional neuroendoscopy: a preclinical laboratory study. World Neurosurg. 2016;92:378-385.

4. Birch K, Drazin D, Black KL, et al. Clinical experience with a high definition exoscope system for surgery of pineal region lesions. J Clin Neurosci. 2014;21(7):1245-1249.

5. Langer DJ, White TG, Schulder M, et al. Advances in intraoperative optics: a brief review of current exoscope platforms. Oper Neurosurg (Hagerstown). 2020;19(1):84-93. 
6. Siller S, Zoellner C, Fuetsch M, et al. A high-definition 3D exoscope as an alternative to the operating microscope in spinal microsurgery. J Neurosurg Spine. 2020;33(5):705-714.

7. Shirzadi A, Mukherjee D, Drazin DG, et al. Use of the video telescope operating monitor (VITOM) as an alternative to the operating microscope in spine surgery. Spine (Phila Pa 1976). 2012;37(24):E1517-E1523.

8. Oertel JM, Burkhardt BW. Vitom-3D for exoscopic neurosurgery: initial experience in cranial and spinal procedures. World Neurosurg. 2017;105:153-162.

9. Beez T, Munoz-Bendix C, Beseoglu K, et al. First clinical applications of a high-definition three-dimensional exoscope in pediatric neurosurgery. Cureus. 2018;10(1):e2108.

10. Mamelak AN, Nobuto T, Berci G. Initial clinical experience with a high-definition exoscope system for microneurosurgery. Neurosurgery. 2010;67(2):476-483.

11. Moisi MD, Hoang K, Tubbs RS, et al. Advancement of surgical visualization methods: comparison study between traditional microscopic surgery and a novel robotic optoelectronic visualization tool for spinal surgery. World Neurosurg. 2017; 98:273-277.

12. Valdés PA, Roberts DW, Lu F-K, Golby A. Optical technologies for intraoperative neurosurgical guidance. Neurosurg Focus. 2016;40(3):E8.

13. Rossini Z, Cardia A, Milani D, et al. VITOM 3D: Preliminary experience in cranial surgery. World Neurosurg. 2017; 107:663-668.

14. Labib MA, Shah M, Kassam AB, et al. The safety and feasibility of image-guided BrainPath-mediated transsulcul hematoma evacuation: a multicenter study. Neurosurgery. 2017; 80(4):515-524.

15. Iyer R, Chaichana KL. Minimally invasive resection of deepseated high-grade gliomas using tubular retractors and exoscopic visualization. J Neurol Surg A Cent Eur Neurosurg. 2018;79(4):330-336.

16. Jackson C, Gallia GL, Chaichana KL. Minimally invasive biopsies of deep-seated brain lesions using tubular retractors under exoscopic visualization. J Neurol Surg A Cent Eur Neurosurg. 2017;78(6):588-594.

17. Bakhsheshian J, Strickland BA, Jackson C, et al. Multicenter investigation of channel-based subcortical trans-sulcal exoscopic resection of metastatic brain tumors: a retrospective case series. Oper Neurosurg (Hagerstown). 2019;16(2): $159-166$

18. Gomez D, Farid S, Malik HZ, et al. Preoperative neutrophilto-lymphocyte ratio as a prognostic predictor after curative resection for hepatocellular carcinoma. World J Surg. 2008; 32(8):1757-1762.

19. Krishnan KG, Schöller K, Uhl E. Application of a compact high-definition exoscope for illumination and magnification in high-precision surgical procedures. World Neurosurg. 2017;97:652-660.

20. Sack J, Steinberg JA, Rennert RC, et al. Initial experience using a high-definition 3-dimensional exoscope system for microneurosurgery. Oper Neurosurg (Hagerstown). 2018; 14(4):395-401.

21. Day JD. Transsulcal parafascicular surgery using Brain Path ${ }^{\circledR}$ for subcortical lesions. Neurosurgery. 2017;64(CN_ suppl_1):151-156.
22. Ritsma B, Kassam A, Dowlatshahi D, et al. Minimally invasive subcortical parafascicular transsulcal access for clot evacuation (Mi SPACE) for intracerebral hemorrhage. Case Rep Neurol Med. 2014;2014:102307.

23. Gonen L, Chakravarthi SS, Monroy-Sosa A, et al. Initial experience with a robotically operated video optical telescopicmicroscope in cranial neurosurgery: feasibility, safety, and clinical applications. Neurosurg Focus. 2017;42(5):E9.

24. Parihar V, Yadav YR, Kher Y, et al. Learning neuroendoscopy with an exoscope system (video telescopic operating monitor): early clinical results. Asian J Neurosurg. 2016; 11(4):421-426.

25. Piquer J, Llácer JL, Rovira V, et al. Fluorescence-guided surgery and biopsy in gliomas with an exoscope system. BioMed Res Int. 2014;2014:207974.

26. Wickham H. ggplot2: Elegant Graphics for Data Analysis. Springer-Verlag; 2009.

\section{Disclosures}

The authors report no conflict of interest concerning the materials or methods used in this study or the findings specified in this paper.

\section{Author Contributions}

Conception and design: Tandon, Raheja, Garg, Katiyar. Acquisition of data: Raheja, Garg, Katiyar, Sharma, Goda. Analysis and interpretation of data: Raheja, Mishra, Katiyar. Drafting the article: Tandon, Raheja, Mishra, Garg, Katiyar, Sharma, Goda. Critically revising the article: Tandon, Raheja, Mishra, Garg, Suri. Reviewed submitted version of manuscript: Tandon, Raheja, Mishra, Sharma, Suri, Kale. Approved the final version of the manuscript on behalf of all authors: Tandon. Statistical analysis: Mishra, Katiyar. Administrative/technical/material support: Tandon, Suri, Kale. Study supervision: Tandon, Mishra, Suri, Kale.

\section{Supplemental Information \\ Videos}

Video 1. https://vimeo.com/480873643.

\section{Online-Only Content}

Supplemental material is available online. Supplemental Questionnaire. https://thejns.org/doi/suppl/ 10.3171/2020.10.FOCUS20786.

Supplemental Tables 1 and 2. https://thejns.org/doi/suppl/ 10.3171/2020.10.FOCUS20786.

\section{Correspondence}

Vivek Tandon: All India Institute of Medical Sciences, New Delhi, India.drtandonvivek@gmail.com. 